\title{
Axial Spondyloarthritis: Current Advances, Future Challenges
}

\author{
Robert D Inman, M.D., FRCPC, FACP, FRCP Edin \\ Schroeder Arthritis Institute, University Health Network and University of Toronto, Toronto, ON, Canada
}

\begin{abstract}
Axial spondyloarthritis (axSpA) is a chronic inflammatory joint disease with a predilection for the spine. It affects young adults and has the potential to have a major impact on quality of life, not only because of the chronic pain and fatigue, but also because of the potential for marked disability related to spinal ankylosis. Early detection of axSpA remains a major challenge, for which there is a heightened sense of urgency since it has been shown that earlier intervention with biologics can alter the progression of radiographic change in the spine. Advances in the genetics of axSpA have highlighted a number of candidate genes conferring susceptibility to the disease, but there is evidence of environmental factors playing a role as well. Recently studies in both clinical and experimental axSpA have implicated alterations in the gut microbiome as playing a key role, and the immunology of the gut-joint axis is becoming better understood. The unmet needs which are shaping the research agenda include improvement in early case identification, sensitive and specific biomarkers which could accurately reflect disease activity and severity, improved understanding of the common pathways of inflammation in the skin, eye and gut in axSpA, and novel therapeutic targets which could have curative potential. (J Rheum Dis 2021;28:55-59)
\end{abstract}

Key Words. Spondylitis, Ankylosing spondylitis, Unmet needs

\section{INTRODUCTION}

Axial spondyloarthritis (axSpA) has a relatively high prevalence in the global population. It also strikes a young adult population, with significant impact on function and quality of life. There is encouraging evidence for disease modification with the biologic agents. Despite these facts, axSpA continue to present significant challenges to clinicians and researchers alike. For clinicians, the changing nomenclature has raised some uncertainties. The term ankylosing spondylitis (AS) was widely accepted and applied both in clinical trials and genetics studies. But the use of the term AS for a clinical entity presupposed radiographic damage to the sacroiliac joints (SIJ) on a pelvic $\mathrm{X}$-ray. It was recognized that having joint damage as a precondition for a diagnosis was too restrictive, and would not apply say, to the diagnosis of rheumatoid arthritis. It was also recognized that there could be a prolonged symptomatic period, perhaps months to years in dura- tion, before diagnostic changes in the SIJ appeared on $\mathrm{X}$-ray. The inclusion of magnetic resonance imaging (MRI) studies in this pre-radiographic phase of the disease has added an additional imaging modality to detect early inflammatory lesions in the SIJ and spine. By convention, axSpA is now subdivided into AS (also referred to as radiographic axSpA) and non-radiographic axSpA (nr-axSpA), the latter being defined by distinctive clinical features and/or MRI evidence of SIJ inflammation. Both clinical subsets of axSpA have the same symptomatic burden of disease, similar extra-articular manifestations, and comparable response to biologic agents. But nr-axSpA is distinguished by a female predominance, as well as a low rate of spinal syndesmophyte formation over time.

Another challenge for clinicians relating to axSpA is the paucity of physical findings, particularly in the early phase of axSpA. While there are defined metrics to measure spinal mobility and aggregate indices (like bath ankylosing spondylitis metrology index [BASMI]), these abnormal-

Received : January 18, 2021, Revised : February 17, 2021, Accepted : March 10, 2021

Corresponding to : Robert D Inman iD http://orcid.org/0000-0002-4750-1422

Schroeder Arthritis Institute, University Health Network and University of Toronto, 399 Bathurst Street 1E-423, Toronto, ON M5T 2S8, Canada. E-mail : robert.inman@uhn.ca

Copyright (c) 2021 by The Korean College of Rheumatology.

This is an Open Access article, which permits unrestricted non-commerical use, distribution, and reproduction in any medium, provided the original work is properly cited. 
ities are seen primarily in advanced disease, by analogy with development of radiographic changes in the spine. In patients whose disease is primarily axial, without associated peripheral arthritis or enthesitis, the physical examination has little discriminant value for the assessment. For many rheumatologists used to assessing disease activity with tender and swollen joint counts, the reliance on subjective patient-reported outcomes (such as the bath ankylosing spondylitis disease activity index) seems somewhat less objective. It has been reassuring that therapeutic trials with TNF inhibitors (TNFi) and IL-17 inhibitors (IL-17i) have shown modification of objective signs of inflammation (C-reactive protein [CRP] and MRI scores). Even more compelling has been the evidence that TNFi slow the rate of radiographic progression in the spine as measured by modified stoke ankylosing spondylitis spine score. An earlier multicenter study from Canada and the US had provided evidence of disease modification [1]. More recently, a large study of a longitudinal observation cohort from Seoul has clearly demonstrated that the rate of radiographic progression was decreased when the patients were on TNFi therapy [2].

For researchers alike, axSpA has presented some distinctive challenges. One conceptual issue is whether axSpA represents an autoimmune disease, an autoinflammatory disease, or something distinctive from either. Autoimmune rheumatic diseases are commonly recognized by distinctive autoantibody profiles, as reflected in systemic lupus erythematosus, rheumatoid arthritis, and ANCA-associated vasculitis. While some studies have indeed demonstrated autoantibodies in axSpA [3], these have shed insights into possible pathogenic mechanisms but have not yet translated into clinical practice. So the older concept of "seronegative" arthritis still persists. But the clinical features of axSpA and the polygenic nature of the disease are quite different from classical autoinflammatory diseases such as familial Mediterranean fever. The strong association with major histocompatibility complex (MHC), a feature shared with many autoimmune diseases, provides indirect evidence that axSpA reflects a disorder anchored in adaptive immunity, albeit without autoantibody formation. The association between human leukocyte antigen (HLA)-B27 and axSpA is the strongest association between all rheumatic diseases and MHC. Another insight from genome-wide association studies in axSpA has been the recognition that polymorphisms in ERAP-1, which also confer susceptibility to axSpA, are seen uniquely in HLA-B27-positive axSpA patients, sug- gesting a gene-gene interaction in disease pathogenesis. Subsequent studies in B27-transgenic mice demonstrated that generation of HLA-B27-presented peptides is distinctively dependent on sculpting of the B27 peptidome by ERAP-1 [4]. Integrity of peptide processing and presentation is of course critical for host immune response to pathogens, and HLA polymorphisms may influence immunity to SARS-coV-2 [5]. It has also been recognized that HLA-B27 not only confers risk for axSpA but also represents the best predictive factor for chronicity in reactive arthritis, a clinical subset of SpA which is defined by a microbial trigger.

In addition to post-dysenteric reactive arthritis, the gut-joint interaction is highlighted by the clinical association of axSpA and inflammatory bowel disease (IBD). A number of longitudinal observation cohorts have reported that $10 \% \sim 15 \%$ of axSpA patients also have IBD, and it has been variable whether the clinical onset of IBD precedes that of axSpA or vice versa. Common genetic susceptibility elements have reinforced this interaction [6]. In addition to symptomatic disease, colonoscopic studies in axSpA patients without gastrointestinal symptoms have reported that up to $60 \%$ have histopathologic evidence of gut inflammation. It is also of interest that in the B27-transgenic model, which recapitulates the spectrum of SpA features, raising such animals in a germ-free environment protects against the characteristic gut, joint and skin inflammation seen with conventional housing [7]. More recent studies from several groups have provided evidence of gut dysbiosis in axSpA patients [8-10], which has been paralleled gut microbiome alteration in animal models $[11,12]$.

The strong association of class I MHC with axSpA has always implicated a possible role for $\mathrm{CD} 8+\mathrm{T}$ cells in in the immunopathogenesis of the disease. Recent studies have examined the role of such cells in shedding light on the important issue of gut-joint trafficking [13]. Since integrins modulate such gut migratory patterns, studies have addressed key integrins in this process. Mature $\mathrm{CD} 8+\mathrm{T}$ cells were found to be enriched in synovial fluid of axSpA patients, with a distinct pattern of integrin expression consisting of $\beta 7, \mathrm{CD} 103, \mathrm{CD} 29$ and CD49a. These integrin-expressing $\mathrm{T}$ cells were more prevalent in axSpA synovial fluid than in rheumatoid arthritis SF controls. RNA-seq analysis of SF-derived CD103+, $\mathrm{CD} 49 \mathrm{a}+, \mathrm{CD} 8+\mathrm{T}$ cells revealed elevated TNFAIP3, GZMB, PRF1, and IL-10. These integrin-expressing $\mathrm{CD} 8+\mathrm{T}$ cells have a transcriptomic and proteomic profile 
which closely mimics similar cells in the gut mucosa. These cells also demonstrate a restricted $\mathrm{T}$ cell receptor profile, which provides indirect support for an antigen-driven process and may shed light on the contribution of class I MHC to SpA pathogenesis. This distinctive integrin-expressing cell population provides a cellular basis for the clinically observed interaction between gut and joint inflammation. Integrins as mediators of this cross-talk have also gained attention through the clinical application of vedolizumab (mAb anti- $\alpha 4 \beta 7$ ) in IBD. There have been reports of exacerbation of axSpA following vedolizumab therapy which might reflect dysregulation of gut-joint trafficking. But it remains unknown how frequently vedolizumab-associated arthritis occurs [14].

In addition to alteration in adaptive immunity, there has been increasing interest in innate lymphoid cells in axSpA. These cells are distinctive in reflecting the cellular basis of innate immunity but share some elements of the adaptive immune response as well. One example are mucosa-associated invariant T (MAIT) cells. These CD8+ T cells have an invariant TCR $\alpha$ profile and recognize antigens presented by the MHC-like molecular MR1. The antigens in this instance are derived from metabolites of vitamin $\mathrm{B} 6$ processed by gut microbes, and the gut microbiome plays an essential role in the development and maturation of MAIT cells. AxSpA patients have been found to have an elevated frequency IL-17A+ MAIT cells in blood compared with healthy controls [15]. There was an enrichment of MAIT cells in SF, which had an exaggerated IL-17 phenotype. IL-17 elevation in AS MAIT cells was dependent on priming with IL-7 but not IL-23 or antigen stimulation. While IL-7R genetic variants have been shown to have an association with axSpA, the implicated IL-7R SNP did no effect on IL-7R expression or function in this study. One notable consequence of this study was to define an IL-23-independent pathway for generation of IL-17. This may shed light on the paradox of IL-17i being effective in axSpA but not IL-23 inhibitors (16).

\section{MAIN SUBJECTS}

\section{The road ahead: challenges and opportunities}

\section{1) Early detection}

AxSpA is still plagued by a prolonged interval, commonly several years, between the onset of symptoms and the diagnosis. This is a source of frustration for patients, who are often referred to a wide array of health care practitioners, and commonly try a broad range of alternative and complementary therapies, before seeing a rheumatologist. There are several factors contributing to this inordinate delay. Chronic low back pain has a high prevalence in the population at large, of which axSpA represents only a small fraction [17]. The clinical differentiation between mechanical back pain and inflammatory back pain presents a challenge for clinicians since no single variable (e.g., morning stiffness) has high discriminant power. Physical examination measures (e.g., BASMI) have been established to monitor disease progression in axSpA cohorts, not to differentiate axSpA from degenerative disc disease, the latter being the main contributor to mechanical back pain. Conventional radiographic studies of SI joints and spine are commonly non-informative in shorter duration axSpA, and the modified New York criteria for AS are weighted toward longer duration disease since structural damage is a prerequisite. The effective utilization of MRI to detect inflammatory lesions before diagnostic radiographic changes are evident has made a substantial contribution to shortening the interval time from symptom onset to diagnosis. Indeed newer imaging modalities, such as low-radiation computer tomography scans, may contribute further to this. Yet much of the delay is incurred prior to the assessment by the rheumatologist, and timely referral for a rheumatology consultation itself needs an improved model of care. One approach is to develop an effective screening mechanism with trained health care professionals, which can function as a triage mechanism to stratify the large low back pain population into axSpA risk categories [18]. Such programs will need further studies on how to generalize this model into larger populations in different health care systems.

\section{2) Biomarker identification}

Biomarkers which have discriminant capacity to differentiate axSpA from mechanical back pain could contribute to shortening the interval to diagnosis, particularly if such a marker can be scaled appropriately, and conducted in a cost-effective manner. At present CRP and ESR are commonly used in this setting but both are lacking in sensitivity and specificity to function as a broad screening instrument. Secondly, biomarkers which reflect ongoing inflammation or incipient new bone formation with fidelity would have value in defining prognosis more accurately than is possible at present. Amongst the nr-axSpA patients there is a subset who will progress to AS over 
time. Amongst the patients with diagnostic sacroiliitis there is a subset who will progress to spinal syndesmophyte formation over time. In both cases, biomarkers which are predictive of such disease progression could equip clinicians with evidence-based decision making on prognosis and therapeutics. This would be a first step toward personalized management of axSpA patient. To identify biomarkers which accurately reflect disease activity and severity will require large, well-phenotyped cohorts with multi-omic technologies applied to longitudinal biospecimens. It is encouraging that several such prospective axSpA cohorts are being developed in several countries.

\section{3) Extra-articular manifestations (EAM)}

The frequency of IBD, acute anterior uveitis (AAU), and psoriasis in axSpA patients presents both an opportunity and a challenge. Inflammation in the gut, eye, and skin which is concomitant with joint inflammation affords the opportunity to probe the genetic and immune commonalities which underlie the attack on these different target organs. The challenge is to apply such knowledge of the shared biology in these disease processes for early detection and timely intervention. Finding the immune signatures which predict EAM, or reflect activity of EAM, would allow for more effective co-management of such patients with the gastroenterologist, ophthalmologist and dermatologist. This combined multidisciplinary approach to comprehensive management of the axSpA patient has particular relevance since the next generation biologics have differential target organ specificities, necessitating a team approach for optimal patient outcomes.

\section{4) New therapeutic targets}

Recently there have been significant technical advances in molecular biology and immunology, such as mass cytometry, miRNA bioprofiling, single-cell RNA sequencing, CRISPR-Cas9, as well as machine learning applied to large genomic, transcriptomic and proteomic data sets. These new techniques open the door to more in-depth insight into the biological basis of axSpA than has heretofore been possible. But more pragmatically, these approaches can identify novel druggable targets that might have significant potential not only for controlling inflammation but also for preventing the osteoproliferation, which is the hallmark of the disease. Recent examples of such translational research include macrophage migration inhibitory factor [19], TYK2 [20], and STAT3
[21]. Ultimately these methodologies could realize the goal of precision medicine for axSpA, whereby the distinctive bioprofile of each patient is integrated with the clinical profile of that patient to design treatment which is specific, targeted, and curative.

\section{CONCLUSION}

Solving the riddle of this ancient disease means overcoming significant hurdles: the slow and heterogeneous natural history of AS, the inaccessibility of the target tissues, and the absence of autoantibodies. But AS quintessentially represents a dynamic interplay of genetic susceptibility and environmental triggers. The tools are now at hand in immunology and genomics to finally put to rest this chronic disabling disease through novel therapeutic targets.

\section{CONFLICT OF INTEREST}

No potential conflict of interest relevant to this article was reported.

\section{REFERENCES}

1. Haroon N, Inman RD, Learch TJ, Weisman MH, Lee M, Rahbar MH, et al. The impact of tumor necrosis factor $\alpha$ inhibitors on radiographic progression in ankylosing spondylitis. Arthritis Rheum 2013;65:2645-54.

2. Koo BS, Oh JS, Park SY, Shin JH, Ahn GY, Lee S, et al. Tumour necrosis factor inhibitors slow radiographic progression in patients with ankylosing spondylitis: 18-year real-world evidence. Ann Rheum Dis 2020;79:1327-32.

3. Tsui FW, Tsui HW, Las Heras F, Pritzker KP, Inman RD. Serum levels of novel noggin and sclerostin-immune complexes are elevated in ankylosing spondylitis. Ann Rheum Dis 2014;73:1873-9.

4. Akram A, Lin A, Gracey E, Streutker CJ, Inman RD. HLA-B27, but not HLA-B7, immunodominance to influen$\mathrm{za}$ is ERAP dependent. J Immunol 2014;192:5520-8.

5. Tavasolian F, Rashidi M, Hatam GR, Jeddi M, Hosseini AZ, Mosawi SH, et al. HLA, immune response, and susceptibility to COVID-19. Front Immunol 2021;11:601886.

6. Danoy P, Pryce K, Hadler J, Bradbury LA, Farrar C, Pointon $\mathrm{J}$, et al. Association of variants at 1q32 and STAT3 with ankylosing spondylitis suggests genetic overlap with Crohn's disease. PLoS Genet 2010;6:e1001195.

7. Taurog JD, Richardson JA, Croft JT, Simmons WA, Zhou M, Fernández-Sueiro JL, et al. The germfree state prevents development of gut and joint inflammatory disease in HLA-B27 transgenic rats. J Exp Med 1994;180:2359-64.

8. Yin J, Sternes PR, Wang M, Song J, Morrison M, Li T, et al. Shotgun metagenomics reveals an enrichment of potentially cross-reactive bacterial epitopes in ankylosing spon- 
dylitis patients, as well as the effects of TNFi therapy upon microbiome composition. Ann Rheum Dis 2020;79:132-40.

9. Tito RY, Cypers H, Joossens M, Varkas G, Van Praet L, Glorieus E, et al. Brief Report: Dialister as a microbial marker of disease activity in spondyloarthritis. Arthritis Rheumatol 2017;69:114-21.

10. Breban M, Tap J, Leboime A, Said-Nahal R, Langella P, Chiocchia $\mathrm{G}$, et al. Faecal microbiota study reveals specific dysbiosis in spondyloarthritis. Ann Rheum Dis 2017;76: 1614-22.

11. Asquith M, Davin S, Stauffer P, Michell C, Janowitz C, Lin P, et al. Intestinal metabolites are profoundly altered in the context of HLA-B27 expression and functionally modulate disease in a rat model of spondyloarthritis. Arthritis Rheumatol 2017;69:1984-95.

12. Chriswell ME, Kuhn KA. Microbiota-mediated mucosal inflammation in arthritis. Best Pract Res Clin Rheumatol 2019;33:101492.

13. Qaiyum Z, Gracey E, Yao Y, Inman RD. Integrin and transcriptomic profiles identify a distinctive synovial CD8+ T cell subpopulation in spondyloarthritis. Ann Rheum Dis 2019;78:1566-75.

14. Varkas G, Thevissen K, De Brabanter G, Van Praet L, Czul-Gurdian F, Cypers H, et al. An induction or flare of arthritis and/or sacroiliitis by vedolizumab in inflammatory bowel disease: a case series. Ann Rheum Dis 2017;76: 878-81.

15. Gracey E, Qaiyum Z, Almaghlouth I, Lawson D, Karki S, Avvaru N, et al. IL-7 primes IL-17 in mucosal-associated in- variant T (MAIT) cells, which contribute to the Th17-axis in ankylosing spondylitis. Ann Rheum Dis 2016;75:2124-32.

16. Baeten D, Østergaard M, Wei JC, Sieper J, Järvinen P, Tam LS, et al. Risankizumab, an IL-23 inhibitor, for ankylosing spondylitis: results of a randomised, double-blind, placebo-controlled, proof-of-concept, dose-finding phase 2 study. Ann Rheum Dis 2018;77:1295-302.

17. O'Shea FD, Boyle E, Salonen DC, Ammendolia C, Peterson $\mathrm{C}, \mathrm{Hsu} \mathrm{W}$, et al. Inflammatory and degenerative sacroiliac joint disease in a primary back pain cohort. Arthritis Care Res (Hoboken) 2010;62:447-54.

18. Passalent L, Hawke C, Lawson DO, Omar A, Alnaqbi KA, Wallis D, et al. Advancing early identification of axial spondyloarthritis: an interobserver comparison of extended role practitioners and rheumatologists. J Rheumatol 2020;47: 524-30.

19. Ranganathan V, Ciccia F, Zeng F, Sari I, Guggino G, Muralitharan J, et al. Macrophage migration inhibitory factor induces inflammation and predicts spinal progression in ankylosing spondylitis. Arthritis Rheumatol 2017;69:1796-806.

20. Gracey E, Hromadová D, Lim M, Qaiyum Z, Zeng M, Yao Y, et al. TYK2 inhibition reduces type 3 immunity and modifies disease progression in murine spondyloarthritis. J Clin Invest 2020;130:1863-78.

21. Jo S, Won EJ, Kim MJ, Lee YJ, Jin SH, Park PR, et al. STAT3 phosphorylation inhibition for treating inflammation and new bone formation in ankylosing spondylitis. Rheumatology (Oxford) 2020 Nov 25 [Epub]. 